\title{
LONG-TERMED VARIABILITY IN THE FAR ULTRAVIOLET FLUX OF Be STARS
}

G. J. Peters

Space Sciences Center, University of Southern California, Los Angeles, CA 90089-1341; USA

R. S. Polidan

Lunar and Planetary Laboratory, University of Arizona, Tucson, AZ 85721; USA

\section{INTRODUCTION}

Optical light variability appears to be commonplace in Be stars, on time scales from days to decades, but the phenomenon has yet to be satisfactorily explained. Some possible causes include changes in 1) the star's photospheric temperature or radius, or 2) the envelope's opacity or continuous emission. To investigate the nature of the photometric variability in Be stars, we have been monitoring the FUV flux of selected objects with the ultraviolet spectrometers (UVS) on the Voyager 1 and 2 spacecrafts (cf. Broadfoot, et al. 1977, and Holberg, et al. 1982, respectively, for information on the instrumentation and calibration, and Drilling, et al. 1984 and Polidan, et al. 1987 for a discussion of data reduction techniques), and report the initial results from these observations in this paper. Stars earlier than B8 radiate $30-$ $70 \%$ of their flux in the region covered by the Voyager UVS (900 - $1700 \mathrm{~A}$, resolution $\cong 15 \mathrm{~A}$ ); both the flux peak and the continuum shortward of the peak are observed. Since the FUV flux distribution and intensity in a $B$ star are quite sensitive to the star's $T$, Voyager observations allow one to confidently assess whether temperature variations are indeed responsible for the observed photometric changes and determine the value of $\Delta \mathrm{T}$ if appropriate.

\section{RESULTS}

a Eri, B4V(e), was observed eleven times during $1979-1980$. The data reveal a $30 \%$ variation in the flux in the band $950-1150 \mathrm{~A}$ (Polidan, et al. 1985). In the case of $\alpha$ Eri, there is compelling evidence that a change in the star's photospheric temperature caused the flux variability, since the ratio $\mathrm{F}_{\min } / \mathrm{F}_{\max }$ shows a well-defined, steady decrease toward shorter wavelengths. ${ }^{2}$ A grid of interpolated Kurucz (1979) mode1 atmospheres suggest that the magnitude of $\Delta T$ is about $750 \mathrm{~K}$.

$66 \mathrm{Oph}$, B2IV-Ve, displays a highly variable, perhaps quasi-periodic, stellar wind (Grady, et a1. 1986). We observe a $25 \%$ variation in the FUV flux and, in Figure 1 , show the flux distribution observed at maximum and minimum FUV 1ight. Five Voyager observations from 1984 February 27 to 1985 April 4 suggest that the FUV flux level is anticorrelated with the strength of the C IV resonance 1ine, which is formed in the wind. In 1985 April, C IV absorption was the weakest observed with IUE $(0.45 \mathrm{~A})$ during the period of our Voyager observations, while in 1984 September it was the strongest $(\sim 2 . \overline{6 \mathrm{~A})}$. The interpretation of the observed flux variations is less certain than in the case of a Eri. Considering the 
statistical and systematic errors in the data from the long wavelength channel, we cannot rule out the possibility that the flux in the region 1300 - 1700 A was invariant. If we fit Kurucz models to data shortward of $1200 \mathrm{~A}$ only, we obtain a $\Delta \mathrm{T}$ of $1000 \mathrm{~K}$.

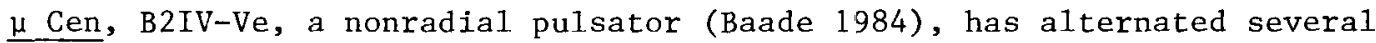
times during the past decade between periods of Balmer emission presence and absence (cf. Peters 1984, 1986, and references therein). Recently, the abrupt onset of $\mathrm{H} \alpha$ emission in just two days was observed (Peters 1986) along with rapid variability in the profile of He I 6678. Three Voyager observations from 1984 June 5 to 1985 June 28 revealed a 25\% variation in the FUV flux in this star (cf. Figure 2). When Ha emission was conspicuous (1.75I) in 1984 June (Peters 1986), the FUV flux was depressed, while in 1985 June, when Balmer emission was absent but nonradial pulsations were prominent, the FUV flux level was elevated. Observations in 1985 May showed a FUV flux comparable with the one seen a month later. As in the case of $66 \mathrm{Oph}$, the flux longward of $1300 \mathrm{~A}$ remained statistically constant during our observations. The flux ratio $\mathrm{F}_{\min } / \mathrm{F}_{\max }$ as a function of wavelength implies that the photospheric temperature was $1000 \mathrm{~K}$ higher during the period of "quiescence" (Balmer emission absent).

\section{CONCLUSIONS}

We have found that the far ultraviolet flux in the three Be stars discussed above varied by $25-30 \%$ on a time scale of months. As the spectral region covered by the Voyager instrumentation contains 40 $50 \%$ of the flux in these stars, these variations involve a sizeable amount of energy (a few hundred solar luminosities). A reasonable interpretation for the flux variability in a Eri is that the star's photospheric temperature changed by $750 \mathrm{~K}$. For $66 \mathrm{Oph}$ and $\mu \mathrm{Cen}$, a similar conclusion is less certain. The signatures of "activity" in the latter two stars appear to be anticorrelated with the level of the FUV flux. Observations of Be stars will continue with the Voyager UVS in order to further investigate the cause for the flux variability, how the FUV flux level correlates with various spectral changes, and the degree to which the FUV flux changes correlate with photometric variations in the optical region.

\section{REFERENCES}

Baade, D. 1984, Astr.Ap., 135, 101. Broadfoot, A. L., et al. $1 \overline{977}$, Space Sci. Rev., 21, 183.

Drilling, J. S., Holberg, J. B., and Schoenberner, D. 1984, Ap.J.,283, L67. Grady, C. A., et al. 1986, preprint.

Holberg, J. B., Forrester, W. T., Schemansky, D. E., and Barry, D. C. 1982, Ap.J., 257, 656.

Kurucz, R. L. 1979, Ap.J.Supp1., 40, 1.

Peters, G. J. 1984, Pub.A.S.P., 96, 960.

Peters, G. J. 1986, Ap.J., 301, L $\overline{61}$.

Polidan, R. S., Peters, G. J., and Stalio, R. 1985, I.B.V.S., No. 2803.

Polidan, R. S., Stalio, R., and Peters, G. J. 1987, Ap.J., in press. 
Figure 1 - The far ultraviolet flux of 66 oph observed with the Voyager UVS during epochs of maximum and minimum FUV light.

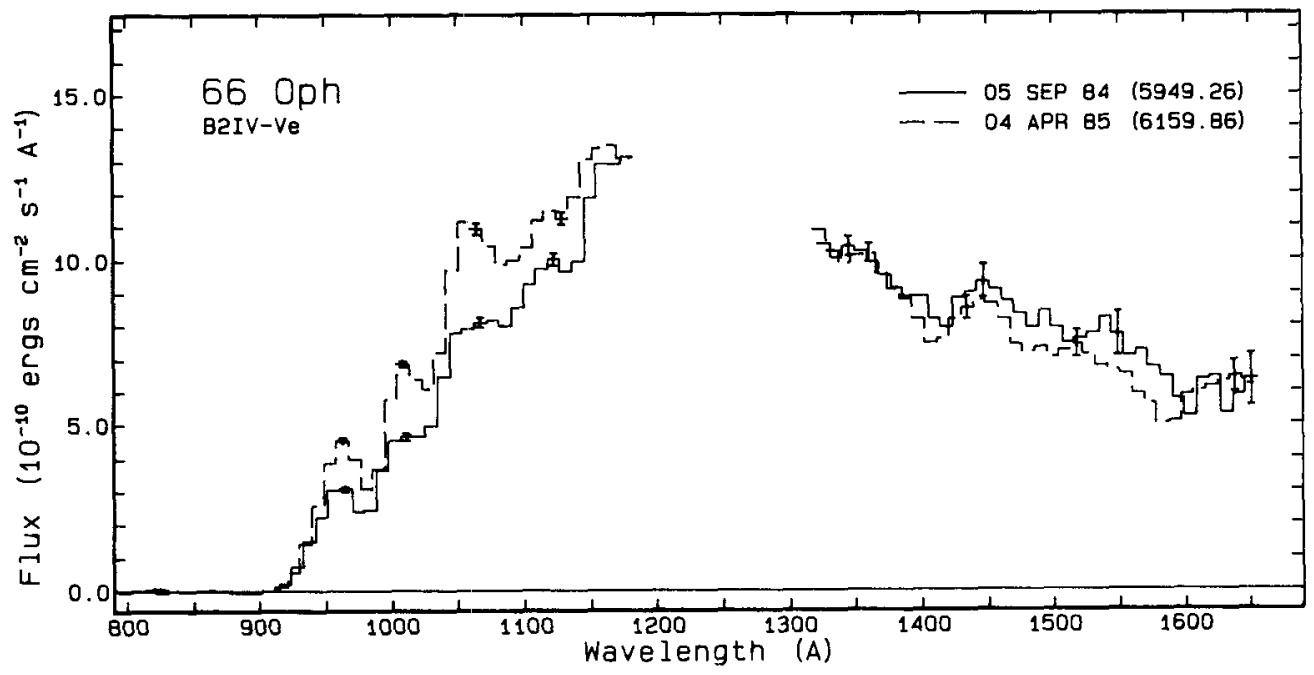

Figure 2 - Same display as in the figure above but for $\mu$ Cen.

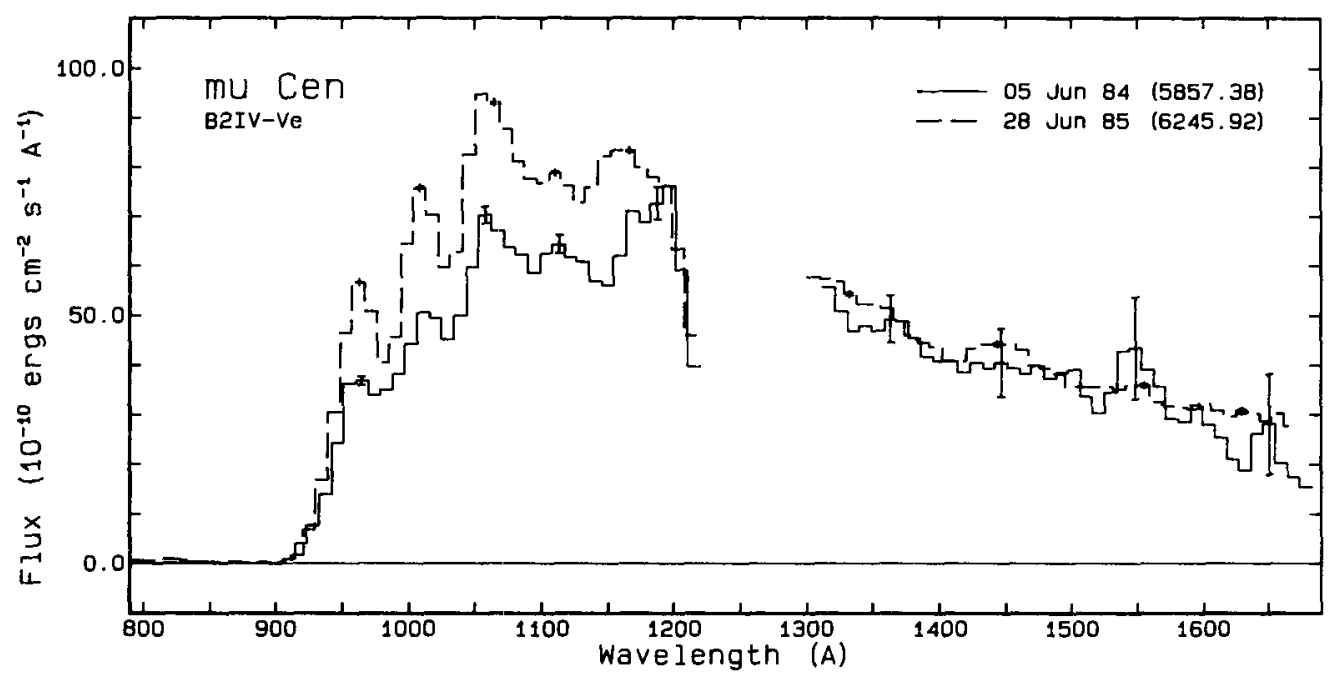




\section{DISCUSSION FOLLOWING PETERS}

Doazan:

I would like to recall that our analysis of the light variations of 88 Her from the far UV to the visual over a decade and through a transition from quasi-normal $B$ to shell phase shows that the appearance of a shell phase is associated with a large luminosity drop in all wavelengths, which implies a drop in $\mathrm{T}_{\text {eff }}$ of about $1000 \mathrm{~K}$. Therefore, the results on temperature changes that you obtain confirm that luminosity changes in Be stars are not only due to changes in the opacity of the outer atmosphere, as has been previously thought, but that a more profound change in the thermodynamic state of the photosphere also occurs.

Peters:

I'm inclined to support your comment. However, AU Mon (an interacting binary Be star) behaves similarly to $88 \mathrm{Her}$, and in the case of AU Mon we currently believe that it is a change in both radius and photospheric temperature that causes the observed long-term flux variability. 